\title{
Pedagogía del dato: perspectiva desde la enseñanza de la estadística en la sociedad del dato*
}

\author{
Giovanni Martínez López \\ Wilson Hernando Soto Urrea****
}

Recibido: 30 de abril de 2018 - Aprobado: 20 de junio de 2018

\section{Resumen}

La omnipresencia del dato en toda actividad humana, la sinergia de diversas disciplinas y el fenómeno de los big data han configurado la sociedad del dato, lo cual implica retos para la formación estadística en el siglo XXI. Si bien la investigación en educación estadística presenta registros de discusiones alrededor de cuáles deberían ser las metas en los diferentes niveles educativos, los resultados no se traducen en las prácticas docentes, entre otras razones, porque la enseñanza de la estadística sigue estando a la sombra de la matemática.

A partir de una exposición acerca de la sociedad del dato se echa una mirada a la investigación sobre alfabetización estadística y pensamiento estadístico, dos de los constructos más explorados por los investigadores y que garantizan que el ser humano pueda desempeñarse como un eficaz prosumidor de datos. Finalmente, se formulan algunas cuestiones que pueden orientar la reflexión de los docentes de estadística a nivel universitario sobre sus prácticas.

\footnotetext{
* Artículo de investigación. Hace parte de la tesis doctoral Sociedad del dato: retos para la formación estadística del profesional del siglo XXI, elaborada en el marco del Doctorado en Educación de la Universidad Santo Tomás. Realizado con el apoyo de la Universidad Católica de Colombia. Citar como Martínez, G. y Soto, W. H. (2019). Pedagogía del dato: perspectiva desde la enseñanza de la estadística en la sociedad del dato. Análisis, 51(94), 141-158. DOI: https://doi. org/10.15332/10.15332/s0120-8454.2019.0094.07

** Matemático de la Universidad Nacional de Colombia y magíster en Docencia e Investigación Universitaria de la Universidad Sergio Arboleda. Docente de tiempo completo de la Universidad Católica de Colombia. Bogotá, Colombia. Correo electrónico: gmartinezl@ucatolica.edu.co. Orcid: https://orcid.org/0000-0002-7693-9829

*** Ingeniero mecánico de la Universidad Nacional de Colombia, magíster en Educación de la Universidad Pedagógica Nacional, doctor en Educación de la Universidad Pedagógica Nacional. Posdoctorado en Educación, Ciencias Sociales e Interculturalidad. Bogotá, Colombia. Correo electrónico: wilsonsoto@usantotomas.edu.co. Orcid: https://orcid. org/0000-0002-0139-0544
} 
Palabras clave: dato, sociedad del dato, prosumidor de datos, alfabetización estadística, pensamiento estadístico. 


\title{
Data pedagogy: a view from statistics teaching in data society*
}

\author{
Giovanni Martínez López** \\ Wilson Hernando Soto Urrea****
}

Data society is the result of the omnipresence of data in every human activity, the synergy of different disciplines and the big data phenomenon, what implies faces for statistical formation in $21^{\text {st }}$ century. Although research into statistical education has discussed about which objectives should be obtained depending on the educational level, results of them have not translated to teaching practices, among other reasons, because teaching statistics is still under the shadow of teaching mathematics.

From an exposition about data society, this article looks at research into statistical teaching, with regard to statistical literacy and statistical thinking, basic elements for the human of $21^{\text {st }}$ in order to be an effective data prosumer. Finally, some questions are formulated in order to guide university teacher's reflection about their practices.

* Research article as a part of the doctoral thesis "Data society: faces to statistical formation for the 21 st century professional" into the Doctoral Degree in Education at Universidad Santo Tomás. Made by the support of Universidad Católica de Colombia. Quote as Martínez, G. y Soto, W. H. (2019). Pedagogía del dato: perspectiva desde la enseñanza de la estadística en la sociedad del dato. Análisis, 51(94), 141-158. DOI: https://doi.org/10.15332/10.15332/s0120-8454.2019.0094.07

** Mathematician at Universidad Nacional de Colombia and Master's Degree in University Teaching and Research at Universidad Sergio Arboleda. Full-time teacher at Universidad Católica de Colombia. Bogotá, Colombia. E-mail: gmartinezl@ucatolica.edu.co. Orcid: https://orcid.org/0000-0002-7693-9829.

*** Mechanical Engineer at Universidad Nacional de Colombia, Master's Degree in Education at Universidad Pegaógica Nacional, Ph.D. Education at Universidad Pegagógica Nacional. Postdoctoral Degree in Education, Social Sciences and Interculturality. Bogotá, Colombia. E-mail: wilsonsoto@usantotomas.edu.co. Orcid: https://orcid. org/0000-0002-0139-0544.4 
Keywords: data, society data, prosumer data, statistical literacy, statistical

thinking. 


\section{Pédagogie des données: une analyse à partir de l'enseignement de la statistique dans la société des données*}

Giovanni Martínez López

Wilson Hernando Soto Urrea**

\section{Résumé}

L'omniprésence des donnés dans toutes les activités humaines, la synergie des disciplines et le phénomène des big data ont donné lieu à une société des données qui implique des défis pour l'enseignement de la statistique au XXI siècle. Bien que la recherche sur l'enseignement de la statistique se questionne sur quels doivent être ses buts, ces recherches ne se traduisent pas en pratiques pédagogiques, et ce parce qu'un tel enseignement reste à l'ombre des mathématiques.

À partir d'une réflexion sur la société des donnés, on présente ici une analyse de l'enseignement de la statistique et de la pensée statistique, deux outils qui garantissent à l'être humain de pouvoir devenir un prosommateur de données. Finalement, on formule quelques questions qui peuvent orienter les enseignants de la statistique.

Mots clés: donnée, société des données, prosommateur de données, enseignement de la statistique, pensée statistique.

\footnotetext{
* Article de recherche élaboré dans le cadre d'une thèse intitulée: Société des données: défis pour la formation statistique au XXle siècle, réalisé à l'Universidad Santo Tomás. Cet article a bénéficié du soutien de l'Universidad Católica de Colombia. Pour citations: Martínez, G. y Soto, W. H. (2019). Pedagogía del dato: perspectiva desde la enseñanza de la estadística en la sociedad del dato. Análisis, 51(94), 141-158. DOI: https://doi.org/10.15332/10.15332/s0120-8454.2019.0094.07

** Mathématicien de l'Universidad Nacional de Colombia y master en Enseignement et recherche de l'Universidad Sergio Arboleda. Professeur à l'Universidad Católica de Colombia. Bogotá, Colombia. Adresse électronique: gmartinezl@ ucatolica.edu.co. Orcid: https://orcid.org/0000-0002-7693-9829

*** Ingénieur mécanique de l'Universidad Nacional de Colombia, master en Éducation de I'Universidad Pedagógica Nacional, docteur en Éducation de l'Universidad Pedagógica Nacional. Pos-docteur en Éducation, Sciences Sociales e Interculturalité. Bogotá, Colombia. Adresse électronique: wilsonsoto@usantotomas.edu.co. Orcid: https://orcid. org/0000-0002-0139-0544
} 


\section{Introducción}

El rol del dato en la historia de la humanidad nunca fue secundario y se ha hecho cada vez más importante en toda actividad humana. Desde los primeros censos poblacionales, que datan de antes de Cristo, hasta el día de hoy, con el fenómeno de los big data ${ }^{3}$, el dato fue no solamente una forma de registro, sino una fuente de información imprescindible y determinante en el desarrollo de la historia. Los censos no solo se hacían con el objetivo de llevar un registro del tamaño de las poblaciones, sino que fueron el insumo para tratar asuntos estatales. Ejemplo de esto fue cuando a finales del siglo XVII William Petty propuso un enfoque cuantitativo para abordar asuntos económicos (Alfaro, Meseguer, Mondéjar y Vargas, 2011).

Con sus trabajos sobre la población del Reino Unido a finales del siglo XVII Petty es reconocido como el pionero de la aritmética política, primera versión de lo que hoy es la estadística. Los siglos siguientes serían testigos del desarrollo de la estadística, palabra que se usó por primera vez para designar "una aglomeración de historias constitucionales, algo de economía política y descripción de las constituciones de los países" (Ferreiro y Fernández, 1988, p. 2) y la necesidad del dato en otros contextos. En el siglo XVIII, con la Revolución industrial, el uso del dato se extendió al contexto del control de la calidad. En el siglo XIX el trabajo con datos experimentales hizo que la estadística se aplicara en la genética, la biología y la agronomía. En el siglo XX el trabajo con datos científicos y las primeras simulaciones con muestras pequeñas hicieron que la estadística se consolidara como ciencia (Yañez, 2000).

La tecnología, como el combustible que impulsa la generación de datos, cada vez más incontrolable en velocidad y tamaño, sumada al nacimiento de un campo emergente como la ciencia de los datos, entre otros aspectos, hacen pensar que la sociedad del siglo XXI es la del dato. Actualmente la presencia y necesidad del dato es inevitable en toda actividad humana, "estamos en una economía digital donde los datos son más valiosos que nunca. Esta es la clave para que todo funcione adecuadamente, desde el gobierno hasta las compañías locales. Sin estos, el progreso se detendría" (Toonders, 2014).

Este artículo es parte de la tesis doctoral "Sociedad del dato: retos para la formación estadística del profesional del siglo XXI". Se trata de una investigación cualitativa con enfoque interpretativo, orientada a revisar el modo como se concibe y se lleva a la práctica la enseñanza de la estadística en pregrados profesionales. Se explicará por qué el siglo XXI es la época de la sociedad del dato, cómo es esta forma de entender el mundo, sus implicaciones para el ser humano y su rol como prosumidor de datos. Posteriormente se revisará cómo las investigaciones en enseñanza de la estadística han abordado esta a través

3 Según Paredes-Moreno (2015) big data es la expresión con la cual se ha identificado a los datos que no pueden ser procesados utilizando la tecnología existente debido a la variedad de formatos en los que se presentan, su cantidad, la velocidad con la que se generan y la incertidumbre con la que suelen ser presentados. 
de los constructos alfabetización estadística (AE) y pensamiento estadístico (PE), factores clave en la formación de ciudadanía estadística (Gould, 2010). Al final la atención se centrará en examinar el estado de la enseñanza de la estadística en Colombia a nivel de educación superior, planteando cuestionamientos que pretenden estimular la reflexión en facultades y departamentos de ciencias básicas para transformar las prácticas docentes en pro de la sociedad del dato.

\section{La sociedad del dato}

El universo es un flujo de datos constante. Ya ni el conocimiento ni la información son la piedra angular de las sociedades, el centro de todo reside en los datos y en cómo procesarlos, adecuándose a la cantidad vertiginosa de ellos que se mueve a través de las redes. Yuval Noah Harari (2015) pone esto a consideración en su libro Homo Deus. El dataísmo, como nueva religión de los datos, une el darwinismo y las formulaciones de la máquina de Turing para llegar a la conclusión de que se pueden aplicar las mismas leyes matemáticas a algoritmos tanto biológicos como electrónicos, por lo que la barrera entre máquinas y animales (incluido el ser humano) se borra. Es decir, todo son datos, y la relevancia de un animal o máquina en el proceso de evolución se debe a su capacidad de procesar su flujo.

Disciplinas como la cibernética, la neurociencia, la matemática y la estadística han alimentado esa nueva forma de concebir el mundo y las relaciones que en este se crean. Norbert Wiener (1998a; 1998b) considera que la comunicación se convierte en el elemento común de todas las disciplinas, por lo que tanto los seres biológicos como los artificiales y mecánicos son entes informacionales. Con esto se cambia el foco de atención de las investigaciones y se desliga a los seres de su diferenciación ontológica. Para Wiener la información que está en medio del proceso comunicativo es el eje de la existencia ya que nada podría ser sin la información y los mensajes que la contienen (Siles, 2007).

La sociedad del dato se nutre de las ideas de Wiener (1998a; 1998b) llevándolas hacia nuevos campos de enunciación, ya que en la actualidad no será la información sino el dato el elemento central. Ello se debe a los avances de la informática, y en específico a los big data, que hacen énfasis en el exuberante acumulado de datos en la red. Hasta 2014 se producía un estimado de 4.4 zetabytes de datos; es decir, 4.4 billones de gigabytes ("Del humanismo al dataísmo", 2016). Quien maneje los datos, maneja al mundo: esa podría ser la premisa que resulta de la comprensión del alcance que aquellos tienen. Sin embargo, no hay un ser (al menos humano) que pueda manejar todos los datos. El sistema es complejo, y por ello, señala Harari (2015), el mercado, que ahora cuenta con el sistema más desarrollado para el procesamiento de los datos, tiene una mano no solo invisible, sino ciega. 
Los desarrollos de la cibernética y la informática han ido de la mano de la neurociencia ya que - gracias al interés de los investigadores por el funcionamiento de los procesos de aprendizaje y conciencia, entre otros - el cerebro se ha convertido en un foco de atención. Para Minsky (1982; 2010), la cibernética ha permitido comprender los procesos cerebrales que lleva a cabo el ser humano. Es por ello que la neurociencia se ha convertido en una disciplina mayor pues hay una relación directa entre el procesamiento de datos de las máquinas y el cerebro del humano.

Las conexiones de la red informática por medio de internet se asemejan a las conexiones neuronales del cerebro. Aunque pueden ser muchas las cualidades de las redes informáticas en cuanto a capacidad de almacenamiento y rapidez del flujo de datos, su actividad se ha basado en el razonamiento humano. Sin embargo, como señala el dataísmo, las máquinas ya no están dirigidas por los humanos, sino orientadas, lo que significa que, aunque hay una programación inicial de la máquina, es esta la que aprende a partir de la corrección de sus propios errores. La máquina, entonces, es consciente de su propio procesamiento, por lo que puede afectar su conducta sin intermediación humana. Ya hacia 1964 Wiener advertía los peligros de la relación entre los hombres y las máquinas cuando afirmaba:

Hoy nosotros somos los creadores, y los robots son nuestros golem. Pero, ¿somos realmente como Dios? Y más aún, ¿cuál es la relación que podemos tener con nuestras criaturas? (Mejor dicho, con nuestras creaciones) ¿Qué responsabilidad tenemos en estos casos?, ¿qué significa, en estos casos, tener la responsabilidad? (Wiener, 1998b)

Si el hombre ya no es el dios de las máquinas, tampoco será el responsable de sus acciones. La autorregulación de aquellas es un gran paso para la neurociencia y para la cibernética ${ }^{4}$, pero las críticas no se han hecho esperar pues la independencia de su procesamiento trae consigo debates de tipo ontológico, axiológico y teleológico. En el plano ontológico, la falta de distinciones entre los seres orgánicos y los no orgánicos sigue siendo un punto de reflexión, principalmente filosófico, religioso y biológico pues el mundo se ha sedimentado en el antropocentrismo y fuera de este muchos paradigmas pierden sentido. Tal es el caso, por ejemplo, de la metafísica sobre la cual se ha fundamentado gran parte de la religión en el mundo: sin la relación estricta entre alma y cuerpo, la persona ya no poseería su rasgo de superioridad frente a animales y máquinas.

4 Cabe recordar que para Wiener la máquina para hacer cálculos se perfeccionaba gracias a la incorporación de las siguientes recomendaciones: "Que el aparato central de adición y multiplicación de la computadora fuera numérico, como en las máquinas corrientes de sumar, en vez de basarse en una medición, como en el analizador diferencial de Bush. Que la regulación de sus mecanismos, que son fundamentalmente dispositivos conectores, se hiciera por medio de tubos electrónicos en vez de engranajes o relés mecánicos, para asegurar una acción más rápida. Que, con arreglo a la política adoptada en algunos aparatos de los laboratorios de la Bell Telephone, sería probablemente más económico un aparato que adoptase la escala dos, por adición y multiplicación, en lugar de la escala diez. Que toda la secuencia de operaciones la compusiera la propia máquina sin que hubiera intervención humana desde la entrada de datos hasta la obtención de los resultados finales, y que todas las decisiones lógicas necesarias para ello las desarrollara la propia máquina. Que la máquina llevara incorporado un aparato para el almacenamiento de datos, que los registrara con rapidez, los mantuviera seguros hasta su cancelación, los leyera deprisa y estuviera inmediatamente listo para almacenar nuevo material" (1998a, p. 26). Es decir, la máquina se autorregularía a partir de la perfección del sistema de flujo de datos pues podría tomar decisiones lógicas con mayor rapidez. 
Ello ha llevado a debates profundos en cuanto al plano axiológico de las máquinas, pues la falta de conciencia podría no ser un problema tan grave como antes se pensaba.

Harari (2015) considera que uno de los problemas que enfrenta la humanidad con el dataísmo es que algoritmos superinteligentes se desliguen de la conciencia. En la actualidad existe un sistema de procesamiento de datos tan complejo (que pareciera no estar manejado por un grupo específico de personas pues gracias a su cúmulo de datos cuenta con una libertad que le permite mover los procesos económicos, políticos y sociales en el mundo), que el hecho de que no sea consciente de la misma humanidad que lo creó podría generar la extinción del Homo sapiens. En palabras de Harari:

Cuando los humanos perdamos nuestra importancia funcional para
la red, descubriremos que, después de todo, no somos la cúspide
de la creación. Las varas de medir que nosotros mismos hemos
consagrado nos condenarán a sumarnos en el olvido a los mamuts
y a los delfines fluviales chinos. En retrospectiva, la humanidad re-
sultará ser solo una onda en el flujo cósmico de datos. (2015, p. 429)

¿Será posible que las máquinas tengan la capacidad de seguir en funcionamiento después de una hipotética extinción de los seres humanos? ¿Podrían funcionar, e incluso autorreproducirse, sin la intermediación de esta especie? Esas cuestiones están lejos de ser resueltas, e incluso algunos dirían que están lejos de ser propuestas en un debate serio. Sin embargo, su formulación permite reflexionar sobre el poder de las máquinas, y más aún, el de los datos en la contemporaneidad.

La estadística adquiere un papel importante en la sociedad pues por su mediación se interpretan fenómenos a partir del procesamiento de datos. Se afirma que ya no importan los datos en sí mismos, sino su cantidad, pues el sistema no se mueve según la información que cada individuo cuelga en la red, sino por el mismo hecho de haberla publicado. Por eso para Harari la siguiente es la premisa de nuestros tiempos: "Si experimentas algo, regístralo. Si registras algo, súbelo. Si subes algo, compártelo" (2015, p. 420). La experiencia en sí misma no es valiosa, sino el hecho de haberla compartido, con lo cual se la agrega al flujo de datos en la red.

Aunque eso puede ser cierto, pues existen millones y millones de datos superfluos en la red, que no tienen incidencia en ninguna estructura de la sociedad, también es cierto que sí hay datos que importan, y hay algoritmos aprovechándose de ellos. Facebook, la red social más grande a nivel planetario, genera cada vez algoritmos más y más perfectos que le permiten al mercado conocer los deseos y necesidades de sus usuarios, de modo que pueda discriminar la información que el usuario recibe. Pero no solo el mercado, también la política ha sacado provecho de esto. Basta con mirar el reciente caso de Cambridge Analytica, consultora británica que accedió a los datos de 87 millones de usuarios 
de Facebook con el fin de analizar los rasgos de cada persona para generar una campaña política directa.

Con ello no se quiere decir que los datos sean utilizados solo para la manipulación de las opiniones de las personas. Como comenta Steve Lohr (2015), inventor del término dataísmo, hay usos benéficos de los datos, tal como el del Hospital Emory, en donde las máquinas generan 160000 datos por segundo para medir los cuidados intensivos que se deben aplicar a las personas enfermas. Los big data, continúa Lohr (2015) son una revolución tecnológica pues las decisiones ya no se basan en las experiencias y la intuición, sino en los datos y en su análisis estadístico.

Los datos ya no son simples elementos contenidos en las redes informáticas pues adquieren un papel relevante en la política, la economía y en definitiva en la sociedad. Ya el collar electrónico del que hablaba Deleuze (Sibilia, 2006) no es un elemento externo al ser, sino que ha sido interiorizado. La imbricación entre la máquina y el hombre cada vez es más notable, y es por ello que la sociedad de los datos y el papel de la estadística en esta son temas de vital importancia para la reflexión académica. Puede que la humanidad sea una onda en el fluir de datos; habrá que revisar entonces las implicaciones de la nueva sociedad.

\section{El ser humano como prosumidor de datos}

En términos de la sociedad del dato todo hombre se comporta como productor o consumidor de datos, o en una sola palabra, como prosumidor de datos. Ya sea que se hable de los departamentos nacionales de estadística, los gobiernos, los responsables de los medios de comunicación o cualquier otra persona, nadie escapa a un mundo donde el dato es omnipresente en toda actividad.

Si se considera la postura de la producción de datos, las implicaciones de la sociedad del dato tienen como protagonistas principales a los departamentos nacionales de estadística. La calidad de sus ejercicios estadísticos es un factor que contribuye a una vida mejor. Al menos así lo afirmó la Organización de las Naciones Unidas (ONU, 2015). Adicionalmente, la Organización para la Cooperación y el Desarrollo Económicos (Ocde) ha hecho un llamado a las naciones en vías de desarrollo a guiar una revolución mundial de los datos; esto es, "proporcionar los datos correctos a las personas adecuadas en el momento apropiado y con el formato preciso" (2015, p. 16).

Asimismo, con miras a contribuir al cumplimiento de los objetivos de desarrollo sostenible propuestos por el Programa de las Naciones Unidas para el Desarrollo (PNUD) para 2030, la Ocde ha propuesto crear un índice de capacidad estadística nacional y controlar su comportamiento reconociendo el carácter público de los datos nacionales: "Para aprovecharlos al máximo, los datos utilizados 
para entender, diseñar y seguir el proceso de desarrollo deberían actualizarse y divulgarse lo más ampliamente posible" (Ocde, 2015, p. 17).

Si se trata de la postura del consumo de datos, entonces es pertinente lo señalado por la Organización de las Naciones Unidas para la Educación, la Ciencia y la Cultura (Unesco) cuando planteó que el alfabetismo mediático e informacional (AMI) es indispensable para el ser humano del siglo XXI. Obtenerlo será vital para lograr el cuarto objetivo de la agenda del PNUD mencionada en el párrafo anterior, esto es, lograr una educación de calidad en población joven y adulta. Eso implica el desarrollo de habilidades como el reconocimiento, uso y comunicación de la información en diversos formatos (Unesco, 2011, p. 185), incluida la importancia de interpretar la información encontrada en medios de comunicación o la que surja del estudio de fenómenos de la vida cotidiana (Batanero, Díaz, Contreras y Roa, 2013).

De todos modos, el escenario que plantea la sociedad del dato es aún más complejo. Los datos no solamente surgen de los vastos ejercicios censales que siempre han existido. Ante el advenimiento de las redes sociales y los big data todas las personas también producen datos. La fábrica de producción es la constante actividad humana. No importa si se estudia o se trabaja, si se crea o se copia, un solo segundo de vida es suficiente para generar datos. Entonces, el hombre no es solo productor o consumidor de datos. Su relación con el dato ha cambiado y lo seguirá haciendo. Gould afirma que "los datos se generan a partir de las actividades, objetos, experiencias y relaciones. Pueden ser almacenados, compartidos y transmitidos. Incluyen un contexto, pero crean contexto" (2010, p. 307). Ya no hay distinción entre productores y consumidores. Por tanto, se debe proveer una formación que lleve a las personas a reconocerse como ciudadanos estadísticos, o en resumen, prosumidores de datos.

Siendo el dato un elemento omnipresente en toda actividad humana, y ya que la estadística ha adquirido una importancia cada vez más creciente, hay un problema educativo importante en el marco de la sociedad del dato: se consumen y se producen datos, pero ¿tiene el ciudadano del siglo XXI las competencias necesarias para ser un eficaz prosumidor de datos?

El problema recae en el dominio de la enseñanza de la estadística. Por lo tanto, se hace preciso revisar lo investigado allí. Después de todo, no hay otro lugar desde el cual se haya trabajado tanto con el afán de formar al ser humano como un prosumidor de datos.

\section{Pedagogía del dato desde la enseñanza de la estadística}

La enseñanza de la estadística empezó a ser importante a partir de 1948, gracias a la necesidad identificada por la Unesco de impulsar la formación estadística 
para mejorar los ejercicios de recolección de datos en países en vías de desarrollo (Batanero, 2000). No obstante, la necesidad de mostrar una visión menos determinista de la realidad para formar científicamente a las personas (Fischbein, citado en Batanero, 2000), la tendencia de los medios de comunicación a representar datos derivados de fenómenos económicos, políticos o meteorológicos (Jacobsen, 1989) y la identificación de la labor estadística como un ejercicio cultural (1998) hicieron que el Instituto Internacional de Estadística (ISI, por sus siglas en inglés) asumiera la responsabilidad de extender la formación estadística a toda persona sin importar su nivel de formación.

Como producto de la actividad del ISI, a partir de la década de los ochenta han sido recurrentes las Conferencias Internacionales de Educación Estadística (Icots, por sus siglas en inglés), los Congresos Internacionales de Educación Matemática (Icme) y diferentes mesas redondas. Posteriormente, con la creación de la Asociación Internacional de Educación Estadística (Iase, por sus siglas en inglés), entre otras acciones se logró posicionar a la estadística en los currículos de educación obligatoria y media a nivel mundial. Parte de los resultados de investigadores sobre enseñanza de la estadística ha llevado a que se formulen diversos constructos que encarnan las metas de formación estadística para las personas, más allá del nivel educativo que se considere. Dos de los más utilizados son la alfabetización estadística (AE) y el pensamiento estadístico (PE).

La AE fue definida por primera vez en Wallman (1993), que reconoció la necesidad de las personas de entender y evaluar críticamente los resultados estadísticos que hacen parte de su cotidianidad. Watson (1997) propuso un modelo jerárquico para formar ciudadanos estadísticamente cultos y advirtió que el fin último es asumir posturas críticas sobre la información encontrada en medios de comunicación. Gal (2002) complementa este modelo resaltando la importancia de las disposiciones, aspecto vinculado a las creencias, las actitudes y la capacidad para asumir posturas críticas, como elementos asociados a la formación en AE. Watson (2006) también señala que un aspecto importante es reconocer cómo los datos son producidos. A nivel iberoamericano el término ha sido traducido por Batanero (2000; Batanero et al., 2013) como "cultura estadística". Serradó Bayés (2013) afirma que, en cualquier caso, la AE fue pensada para formar a las personas como buenos consumidores de datos.

Por mucho tiempo parecía haber un consenso en que una meta razonable para la formación en estadística era la AE. En ese sentido, Franklin et al. —en Guidelines for Assesment and Instruction in Statistics Education (Gaise) - planteaban que los cursos de análisis de datos y probabilidad deberían

capacitar a los estudiantes para a) formular preguntas orientadas a la recolección de datos, organizar y mostrar aspectos relevantes de ellos, b) seleccionar y usar métodos apropiados para analizar datos, c) desarrollar y evaluar inferencias y predicciones basadas en los datos y d) entender y aplicar conceptos básicos de probabilidad. (2005, p. 5) 
No obstante, la versión 2016 de Gaise considera factores como el aumento de estudiantes en cursos de estadística a nivel mundial, el surgimiento de la ciencia de los datos como un campo emergente y la exigencia del uso de recursos tecnológicos para justificar la necesidad de enseñar pensamiento estadístico (PE) sin descuidar la necesidad de formar consumidores críticos (esto es, formar en AE).

Cronológicamente el PE fue abordado en forma paralela a la AE. Las primeras definiciones se encuentran en el contexto del control de calidad, pero con el tiempo las investigaciones lo asumieron como otra posible meta de formación. La guía de la American Society for Quality (ASQ) caracteriza al PE con base en los planteamientos de Deming acerca de lo que es el conocimiento profundo de un sistema:

Una filosofía de aprendizaje (una forma de pensar, distante de las técnicas estadísticas) y acción basada en tres principios: a) todo ocurre en un sistema de procesos interconectados, b) la variación existe en todos los procesos y c) comprender y reducir la variación es clave para el éxito. (ASQ, 1996, p. 5)

En el contexto de la educación estadística, Moore (1997) afirmó que es un modo de razonamiento sobre aspectos centrales en estadística - entre ellos el dato, el reconocimiento de su necesidad y la producción - y que sus principios siguen vigentes en los tiempos de los big data, a pesar de que los precursores de estos no los tomen en cuenta (Hoerl, Snee y De Veaux, 2014). Aunque su definición ha sido un tema de discusión, las investigaciones apuntan a caracterizar al pensador estadístico. Wild y Pfannkuch (1999) añaden que maneja distintas formas de representación de los datos (trasnumeración). Chance (2002) menciona que mantiene una actitud escéptica hacia los datos obtenidos, relaciona la información estadística con el contexto sin tecnicismos y piensa más allá de los textos guía, algo ratificado en Behar y Grima (2004). Hoerl y Snee (2010) mencionan que trata con cuidado las fuentes de variabilidad.

El PE suele confundirse con el razonamiento estadístico (RE) en investigaciones en español por malas traducciones de trabajos anglosajones; pero su uso deliberado también ha llevado a que se presenten confusiones entre estos términos. Por largo tiempo fue interés de las comunidades mundiales en educación estadística poner en claro sus diferencias. Snee (1990) menciona que el PE trasciende los textos escolares, a diferencia de los otros constructos. Ito (2000), en referencia a los diversos niveles de formación, mencionó que la AE (entendida como capacidad estadística) debería ser objetivo de la formación obligatoria, media y de cursos introductorios de estadística; el PE, por su parte, debería ser meta para todos los niveles de educación, incluidos futuros analistas de datos y educadores en estadística; mientras que el RE es meta para futuros estadísticos en todos los niveles de formación. DelMas (2002) afirma que la AE es la meta básica para potenciar el PE y el RE. 
La guía Assesment Resource Tools for Improving Statistical Thinking (Artist, 2006) provee recursos para los docentes a fin de entender más claramente estos conceptos. En términos de la evaluación y siguiendo la taxonomía de Bloom, el PE está relacionado con los tres niveles más altos (análisis, síntesis y evaluación); el RE, con el segundo nivel (comprensión), y la AE, con el primer nivel (conocimiento).

\section{¿Hay alfabetización y pensamiento estadístico en la práctica?}

Aunque el interés por los temas expuestos es creciente, aún hay crisis por la forma como se enseña estadística. Por más que la inclusión en currículos de educación básica y media lleve cerca de tres décadas, y que la enseñanza en educación superior data de mucho tiempo atrás, la estadística se enseña aún bajo la sombra de la matemática. Zapata afirma:

Por años, la enseñanza de la estadística se ha desarrollado al amparo de la enseñanza de las matemáticas y por ser las matemáticas una ciencia de naturaleza determinística, la enseñanza de la estadística también ha adoptado este carácter. La estadística en sí misma ostenta una naturaleza no determinística porque la variación es una de sus particularidades. Parece ser que, a pesar de esta peculiaridad, por mucho tiempo, la enseñanza de la estadística ha ignorado su naturaleza. (2011, p. 236)

Enseñar estadística como una rama de la matemática detiene la posibilidad de aprender a analizar datos (Donoho, 2015). Lo anterior no ofrece un panorama alentador sobre los resultados de la enseñanza de la estadística. De acuerdo con Batanero et al.:

La estadística es una disciplina científica autónoma, que tiene sus métodos específicos de razonamiento. Apoyo esta afirmación en el hecho de que la estadística no ha surgido de la matemática, sino de una serie de ciencias que se han apoyado en la matemática. Más aún, la relación entre estadística y matemáticas no es biunívoca; la estadística toma conceptos matemáticos para el desarrollo de sus métodos, en cambio la matemática no usa conceptos estadísticos. La estadística tiene también controversias específicas (por ejemplo, sobre el significado de la probabilidad), y la posición que un estadístico toma sobre ellas tiene un impacto inmediato en su práctica. (2013, p. 2)

Aunque parece haber un consenso en que la AE, el PE y el RE son metas que toda persona debiera alcanzar al final de su formación en estadística, hay más dudas que certezas sobre cómo llevar esto a la práctica y si el establecimiento de estas metas ha dado resultados. Campos (2016) sugiere que por más que se ejecuten 
acciones que puedan estimularlas, ninguna puede ser enseñada directamente en el aula. MacKay (2016) señala que no hay referencias sobre investigaciones que evalúen los niveles de AE o PE en adultos, lo cual no ayuda a establecer si lo que se ha investigado ha tenido efectos o no. Weiland (2017) señala que a nivel de educación en la escuela, por más que se invoque a la AE como parte del currículo en matemáticas, en el mejor de los casos aquella es abordada superficialmente y dando prioridad al manejo algorítmico.

Da la impresión de que cualquier docente que haya tenido una ligera exposición al análisis de datos o al pensamiento determinístico es capaz de asumirlos sin problemas. Además, en el mejor de los casos se cuenta con paquetes de software especializados y textos de estadística que no resultan funcionales. En la práctica, el manejo de software se vuelve meramente instruccional y no siempre se toman en cuenta las disposiciones de los estudiantes hacia su uso (Schuyten y Thas, 2007). Además, según Gould (2010) los textos ofrecen una visión numérica, limitada, poco útil y abstracta acerca del dato, lo cual no ayuda en la formación de competencias para la sociedad del dato.

La situación en las universidades no es distinta. De hecho, es todavía más crítica si se toma en cuenta que la mayoría de investigaciones en enseñanza de la estadística se centran en educación básica y media. Rocha (2013) diagnostica que en las facultades y los departamentos de ciencias básicas hay pocas evidencias de ejercicios reflexivos acerca de cómo se enseña estadística. También advierte otros inconvenientes. Entre los más críticos se encuentran la falta de uso de la estadística entre profesores y estudiantes - “un porcentaje muy bajo de los profesores admiten poseer bases sólidas y actualizadas en métodos y técnicas estadísticas" (2013, p. 35) - , la poca oferta de programas de formación y actualización en estadística y el escaso desarrollo de herramientas para la enseñanza de la estadística.

\section{La enseñanza de la estadística a nivel de educación superior en Colombia}

No hay mención de qué tan importante sería la estadística para la formación de profesionales colombianos, salvo en Rocha (2013), que investigó sobre las prácticas docentes de los profesores de probabilidad y estadística. La Asociación Colombiana de Facultades de Ingeniería (Acofi) plantea que la matemática, las ciencias naturales y las humanidades son ejes transversales en la formación de ingenieros, pero no menciona la estadística. En el caso de la Asociación Colombiana de Facultades de Ciencias Económicas (Afadeco) no existen documentos que hagan alguna referencia a la estadística. La situación no es distinta en el caso de la Asociación Colombiana de Facultades de Psicología (Ascofapsi), algo que puede ser sorprendente ya que - de acuerdo con Ravelo, Mejía y González (2016) - el 87.3 \% de las publicaciones hechas en la revista Acta Colombiana de 
Psicología en el período 2010-2014 (se trata de la revista colombiana de sicología mejor escalafonada en 2016 según Scimago) provienen de estudios cuantitativos.

Quizá la instancia en donde se podría observar resultados de la formación en estadística o alguna mención de su importancia sea las pruebas estatales Saber Pro y Saber TyT, destinadas a evaluar la calidad de la educación superior. Dentro de los módulos que las componen aparece el de razonamiento cuantitativo, entendido como "el conjunto de elementos de las matemáticas (sean estos conocimientos o competencias) que permiten a un ciudadano tomar parte activa e informada en el contexto social, cultural, político, administrativo, económico, educativo y laboral" (Icfes, 2017a, p. 31), que debe ser resuelto por toda persona que presenta la prueba. Otro módulo, aunque no discrecional para todo pregrado, es el de pensamiento científico, entendido como "la capacidad que tienen los estudiantes de comprender, analizar y afrontar situaciones reales o abstractas con rigor científico" (Icfes, 2017b, p. 16), tradicionalmente elegido por pregrados en ingeniería, ciencias naturales, matemáticas y estadística. De todos modos, en ambos módulos la estadística sigue bajo la sombra de la matemática, tendencia que se replica en educación básica y media.

Desde luego, la preocupación por el desempeño en estos módulos es tema de debate en las instituciones de educación superior. Sin embargo, se desconoce si los ítems de estas pruebas que involucran conceptos estadísticos o manejo de datos evalúan $\mathrm{AE}$ o $\mathrm{PE}$, competencias indispensables para las personas de la sociedad del dato, lo que hace más difícil saber cuál es la situación actual de la formación estadística a nivel nacional en el marco de la educación superior.

\section{Conclusiones y preguntas para la discusión}

La sociedad del dato plantea retos para la formación estadística de toda persona, sin importar su modus vivendi: no se requiere trabajar en un departamento de estadística para producir datos, la vida misma se nutre y se alimenta de ellos y todo ser humano asume, consciente o inconscientemente, la responsabilidad de consumir datos. Por eso el ser humano es hoy un prosumidor de datos.

El tiempo es testigo de cómo la percepción del ser humano acerca del dato ha cambiado debido a su necesidad en toda actividad humana. Investigadores en enseñanza estadística y profesores deben estar preparados para enfrentar el reto de estudiantes que han tenido exposición a los datos incluso antes de comenzar sus cursos de estadística (Gould, 2010). No es que todo ciudadano deba convertirse explícitamente en un científico de datos, pero la formación estadística que reciba debe ayudarlo a tomar posturas críticas ante los datos que consume y conciencia ante las implicaciones que tienen sus ejercicios de producción de datos. 
Particularmente en el contexto de la educación superior esto es aún más importante. Todo profesional, en mayor o menor medida, es un futuro analista de datos en el sentido de que debe enfrentarse a situaciones cotidianas o profesionales que involucran consumo y producción de datos. Por tal razón, las metas de AE y PE son aún más urgentes en tales casos.

Lo mostrado en este artículo plantea un escenario de incertidumbre para la enseñanza de la estadística, pero asimismo, mucho trabajo por hacer. ¿Cuáles son los resultados que se están consiguiendo producto de la formación estadística impartida en las universidades? ¿Un buen desempeño en las pruebas estatales garantiza que las personas están siendo formadas adecuadamente para la sociedad del dato? ¿Conseguir tasas bajas de deserción en los cursos de estadística garantizará que los profesionales en formación tendrán las competencias requeridas en la sociedad del dato?

Si el docente es el primer eslabón en la cadena de formación del ser humano como prosumidor de datos, ¿cómo llevar al docente de estadística universitario colombiano a reflexionar sobre el modo como enseña estadística? ¿Cuáles acciones se pueden implementar para mejorar los niveles de AE y PE en docentes universitarios y futuros profesionales?

\section{Referencias}

Alfaro, J., Meseguer, M., Mondéjar, J. y Vargas, M. (2011). Los inicios de la aritmética política en la Inglaterra del siglo XVII. En J. M. Ribóo e I. Ribóo (eds.) Historia de la probabilidad y la estadística V (pp. 1-9). Santiago de Compostela: Asociación de Historia de la Estadística y de la Probabilidad de España.

American Society for Quality Statistics Division (ASQ, 1996). Statistical Thinking. Publicación especial disponible en American Society for Quality, Quality Information Center, Milwaukee, WI.

Artist (2006). Assesment Resource Tools for Improving Statistical Thinking. Recuperado de https://apps3.cehd.umn.edu/artist/about.html

Batanero, C. (2000). ¿Hacia dónde va la educación estadística? Blaix, 15, 2-13.

Batanero, C., Díaz, C., Contreras, J. y Roa, R. (2013). El sentido estadístico y su desarrollo. Números, 83, 7-18.

Behar, G. y Grima, P. (2004). La estadística en la educación superior: ¿Formamos pensamiento estadístico? Ingeniería y Competitividad, 5, 84-90.

Campos, C. R. (2016). La educación estadística y la educación crítica. En I. Álvarez y C. Sua (eds.), Memorias del II Encuentro Colombiano de Educación 
Estocástica (pp. 5-23). Bogotá: Asociación Colombiana de Educación Estocástica.

Chance, B. (2002). Components of statistical thinking and implications for instruction and assessment. Journal of Statistics Education, 10(3).

Del humanismo al dataísmo (13 de septiembre de 2016). Lateralia. Recuperado de https://lateralia.es/del-humanismo-al-dataismo/

DelMas, R. (2002). Statistical literacy, reasoning and learning: A commentary. Journal of Statistical Education, 10(3).

Donoho, D. (2015). 50 years of data science. Journal of Computation and Graphical Statistics, 26(4), 745-766.

Ferreiro, O. y Fernández, P. (1988). La estadística, una ciencia en la controversia. Revista Universitaria, 25. Recuperado de https://goo.gl/5dkB52

Franklin, C., Kader, G., Mewborn, D., Moreno, J., Peck, R. y Perry, M. (2005). Guidelines for Assessment and Instruction in Statistics Education (Gaise) report: A pre-K-12 curriculum framework. Alexandria, VA: American Statistical Association.

Gaise College Report, ASA Revision Commitee (2016). Guidelines for Assesment and Instruction in Statistics Education: College report 2016. Recuperado de http://www.amstat.org/education/gaise

Gal, I. (2002). Adult's statistical literacy: Meaning, components, responsibilities. International Statistical Review, 70(1), 1-25.

Gould, R. (2010). Statistics and the modern student. International Statistics Review, 78(2), 297-315.

Harari, Y. N. (2015). Homo Deus. Bogotá: Penguin Random House.

Hoerl, R. y Snee, R. (2010). Statistical thinking and methods in quality improvement: A look to the future. Quality Engineering, 22(3), 119-129.

Hoerl, R., Snee, R. y De Veaux, R. (2014). Applying statistical thinking to big data problems. WIREs Comput Stat, 6, 222-232.

Instituto Colombiano para la Evaluación de la Educación (Icfes, 2017a). Guía de orientación módulos de competencias genéricas Saber Pro 2017. Bogotá: Icfes. Recuperado de https://goo.gl/ht4eNB

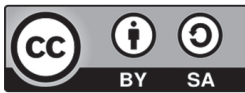

